mutations in mature GCs. Multiple mechanisms, including lack of GLT expression and downregulation of the enzyme APE1, likely a target of the transcriptional repressor BCL-6, were proposed to contribute to the repression of CSR in GC B cells.

Interestingly, in silico analysis of CSR and SHM suggested that the determination of the isotype before the GC phase of intense $B$ cell selection and proliferation promotes isotype diversity, whereas ongoing switching in GCs would homogenize the isotype distribution and would be incompatible with the observation of IgM-dominated GCs. The authors also point out that restricting CSR from GCs reduces the risk of GC B cells carrying pathogenic double-stranded breaks becoming long-lived. It also allows production of IgM memory B cells that can switch to protective isotypes upon encounter with antigenically related pathogens.

Alexandra Flemming

ORIGINAL ARTICLE Roco, J. A. et al. Class-switch recombination occurs infrequently in germinal centers. Immunity https://doi.org/10.1016/j.immuni. 2019.07.001 (2019)

a PLGF-STAT3 axis favours the differentiation of disease-causing $\mathrm{T}_{\mathrm{H}} 17$ cells at the expense of homeostasis-maintaining $\mathrm{T}_{\text {reg }}$ cells. Yoo et al. used models of delayedtype hypersensitivity and experimental autoimmune encephalomyelitis in Plgf-transgenic and Plgf-knockout mice to show that this signalling axis is relevant to $T_{H} 17$ cell-mediated diseases in vivo. Furthermore, they showed that human $T$ cells also produce IL-17 in response to PLGF. In synovial fluid samples of patients with rheumatoid arthritis, levels of PLGF production by $\mathrm{CD}^{+} \mathrm{T}$ cells correlated with levels of IL-17, particularly in patients with low levels of IL- 6 in synovial fluid. This study outlines a positive feedback loop between angiogenesis and autoimmunity mediated by $T_{H} 17$ cell production of PLGF, which might therefore be a new therapeutic target.

Kirsty Minton

ORIGINAL ARTICLE Yoo, S.-A. et al. Placental growth factor regulates the generation of $\mathrm{T}_{\mathrm{H}} 17$ growth factor regulates the generation of $\mathrm{T}_{H} 17$
cells to link angiogenesis with autoimmunity. Nat. Immunol. https://doi.org/10.1038/ s41590-019-0456-4 (2019)

\title{
REGULATORY T CELLS
}

\section{A metabolic peace process}

In humans, loss-of-function mutations in the key regulatory $T\left(\mathrm{~T}_{\text {reg }}\right)$ cell transcription factor FOXP3 lead to the severe autoimmune disease IPEX (imunodysregulation, polyendocrinopathy, enteropathy, $\mathrm{X}$-linked). A recent study suggests that $T_{\text {reg }}$ cell function could be restored in such patients by targeting the mTOR pathway.

Although $\mathrm{T}_{\text {reg }}$ cells still develop in the thymus of FOXP3-deficient patients and mice, they show defective suppressive activity and acquire effectorlike functions. Charbonnier et al. reasoned this could be linked to altered cell metabolism, as effector T cells show several metabolic changes, including an increase in aerobic glycolysis and oxidative phosphorylation (OXPHOS). The authors generated a Foxp $3^{\triangle \mathrm{AEGFPC}}$ knock-in mouse system that allowed for the co-deletion of other molecules in FOXP3-deficient $T_{\text {reg }}$ cells. Similarly to other FOXP3-deficient strains, male mice hemizygous for the Foxp $3^{\Delta E G F P i C r e}$ allele were runted and died early from autoimmune lymphoproliferative disease. Compared with control $T_{\text {reg }}$ cells, the FOXP3deficient $T_{\text {reg }}$ cells from Foxp $3^{\triangle E G F P i C r e}$ mice showed increased activation of the mTOR pathway, which is crucial for supporting effector T cell metabolism and function.

The mTOR kinase is the catalytic subunit of two distinct complexes; mTORC1 and mTORC2. The authors therefore generated mice in which FOXP3-deficient $T_{\text {reg }}$ cells also lacked RAPTOR and/ or RICTOR, which are key components of mTORC1 and mTORC2, respectively. Mice with $\mathrm{T}_{\text {reg }}$ cells deficient in both FOXP3 and RICTOR still showed lymphoproliferation but had less tissue inflammation, increased body weight and better survival compared with mice with FOXP3deficient $T_{\text {reg }}$ cells. By contrast, deletion of RAPTOR or both RAPTOR and RICTOR in FOXP3deficient $\mathrm{T}_{\text {reg }}$ cells did not ameliorate disease. However, mice with RAPTOR-deficient FOXP3deficient $T_{\text {reg }}$ cells showed a reversal of the $T_{\text {reg }}$ cell population expansion seen in Foxp $3^{\Delta E G F P i C r e}$ mice. Therefore, mTORC1 seems to support the population expansion of FOXP3-deficient $\mathrm{T}_{\text {reg }}$ cells in Foxp $3^{\triangle E G F P i C r e}$ mice, whereas $m T O R C 2$ drives the dysregulation of FOXP3-deficient $\mathrm{T}_{\text {reg }}$ cells that causes inflammatory disease.

Indeed, RICTOR deficiency in FOXP3-deficient $\mathrm{T}_{\text {reg }}$ cells restored their suppressor functions and reversed the upregulation in Thelper $1\left(T_{H} 1\right)$ cell-type effector activity. RICTOR deficiency also enhanced regulatory functions in FOXP3-sufficient $T_{\text {reg }}$ cells but did not cause effector $T$ cells to acquire suppressive functions.

Transcriptomics indicated that RICTOR deficiency in FOXP3-deficient $\mathrm{T}_{\text {reg }}$ cells is associated with the upregulation of a core set of $\mathrm{T}_{\text {reg }}$ cell-associated genes, including $l l 10$, and with the suppression of genes linked to effector T cell function. Notably, neutralization of IL-10 abrogated the improved suppressor activity of RICTOR-deficient FOXP3-deficient $\mathrm{T}_{\text {reg }}$ cells. Further experiments indicated that the improved suppressor functions seen in RICTOR-deficient FOXP3-deficient $T_{\text {reg }}$ cells were largely dependent on the activity of FOXO1, which is an important negative regulator of the $T_{H} 1$ cell programme.

Metabolic analyses indicated that FOXP3deficient $\mathrm{T}_{\text {reg }}$ cells had increased expression of enzymes linked with the glycolytic and pentose phosphate pathways and showed an increase in glycolysis and OXPHOS; concurrent RICTOR deficiency reversed most of these changes. Moreover, deletion of the bifunctional enzyme PFKFB3 - a potent stimulator of glycolysis in FOXP3-deficient $\mathrm{T}_{\text {reg }}$ cells had a similar effect to RICTOR deletion in restoring their capacity to suppress $T_{H} 1$ cell-type immune responses, although unlike RICTOR deficiency it did not restore the capacity of FOXP3-deficient $T_{\text {reg }}$ cells to suppress effector T cell proliferation. Loss of both PFKFB3 and RICTOR from FOXP3-deficient $\mathrm{T}_{\text {reg }}$ cells did not have an additive effect, confirming aerobic glycolysis as a common target of both interventions; therefore, mTORC2-dependent metabolic dysregulation, including increases in glycolysis and OXPHOS, may drive distinct facets of the regulatory dysfunction in FOXP3-deficient $\mathrm{T}_{\text {reg }}$ cells.

Importantly, the authors found that $\mathrm{T}_{\text {reg }}$ cells from patients with IPEX also showed increased glycolytic activity. Although $\mathrm{T}_{\text {reg }}$ cells from these patients do not normally suppress effector T cell proliferation in vitro, pretreatment with an mTOR inhibitor enabled this suppressive capacity. Furthermore, mTOR inhibitors were found to heighten the suppressive functions of FOXP3sufficient $\mathrm{T}_{\text {reg }}$ cells from control individuals. Therefore, targeting mTOR could improve $\mathrm{T}_{\text {reg }}$ cell functions not only in patients with IPEX but in patients with other autoimmune or inflammatory diseases not associated with FOXP3 deficiency. Yvonne Bordon

ORIGINAL ARTICLE Charbonnier, L.-M. et al. Functional reprogramming of regulatory T cells in the absence of Foxp3. Nat. Immunol. https://doi.org/10.1038/s41590-019-0442-x (2019) 